\title{
Alcohol use and sexual risk behaviors in the Armed Forces of the Democratic Republic of the Congo
}

Bonnie Robin $\operatorname{Tran}^{1,2^{*}}$ (D), Anthony Davis ${ }^{1,2}$, Margo Sloan ${ }^{1,2}$, Carol Macera ${ }^{1,2}$, Anthony Mutombe Mbuyi ${ }^{3}$ and Gilbert Kurhgnga Kabanda ${ }^{3}$

\begin{abstract}
Background: Alcohol misuse is an important contributor to sexual acquisition and transmission of HIV in military communities. This cross-sectional study quantified the prevalence of probable problematic alcohol use among male service members in the Armed Forces of the Democratic Republic of the Congo (FARDC), identified associated factors, and investigated associations of alcohol misuse with risky sexual behaviors.

Methods: Participants included 2549 active duty male soldiers $\geq 18$ years old. Data were collected via computerassisted personal-interview from October 2013-April 2014. The Alcohol Use Disorders Identification Test (AUDIT) was used to identify probable problematic alcohol use (AUDIT score $\geq 8$ ) compared to no/low-risk alcohol use (AUDIT score $\leq 7$ ). Bivariate logistic regressions were used to identify factors associated with probable problematic alcohol use. Several multivariable logistic regressions (adjusted for age, marital status, education level) were used to examine associations of probable problematic alcohol use with risky sexual behaviors. Tests were two sided; statistical significance was defined as $p<0.05$.

Results: Fifteen percent of men screened positive for probable problematic alcohol use. The odds of probable problematic alcohol use were elevated among men who were single and living with a partner $(\mathrm{OR}=1.66 ; 95 \% \mathrm{Cl}=1.24-2.21)$, ranked as a non-commissioned officer [NCO] (OR $=1.40 ; 95 \% \mathrm{Cl}=1.10-1.77)$, and in the 30-39 and 40-49 age groups (OR 30-39 age group $=2.17 ; 95 \% \mathrm{Cl}=1.56-3.02 ; \mathrm{OR} 40-49$ age group $=1.79 ; 95 \% \mathrm{Cl}=1.26-2.55)$. Probable problematic alcohol use was associated with increased odds of having sex with a sex worker (SW), having multiple sexual partners, and participating in transactional sex (aOR sex with a SW $=2.36 ; 95 \% \mathrm{Cl}=1.78-3.13$; aOR multiple sexual partners $=2.08 ; 95 \% \mathrm{Cl}=1.66-2.60 ; \mathrm{aOR}$ transactional sex $=1.99 ; 95 \% \mathrm{Cl}=1.59-2.50)$.

Conclusions: Results emphasize the need to address alcohol use in the FARDC and integrate alcohol abuse education into HIV prevention programs among male service members. Alcohol abuse prevention efforts should target men who are 30-49years of age, unmarried, and ranked as a NCO. Messages and interventions to reduce alcohol misuse in relation to risky sexual behaviors are needed.
\end{abstract}

Keywords: Alcohol, Sexual risk behaviors, HIV, Military populations

\footnotetext{
* Correspondence: bonnie.r.tran.ctr@mail.mil

${ }^{1}$ Department of Defense HIV/AIDS Prevention Program, San Diego, California,

USA

2Leidos Inc, Reston, Virginia, USA

Full list of author information is available at the end of the article
}

(c) The Author(s). 2019 Open Access This article is distributed under the terms of the Creative Commons Attribution 4.0 International License (http://creativecommons.org/licenses/by/4.0/), which permits unrestricted use, distribution, and reproduction in any medium, provided you give appropriate credit to the original author(s) and the source, provide a link to the Creative Commons license, and indicate if changes were made. The Creative Commons Public Domain Dedication waiver (http://creativecommons.org/publicdomain/zero/1.0/) applies to the data made available in this article, unless otherwise stated. 


\section{Background}

In 2017, UNAIDS reported that approximately 36.9 million [31.1-43.9 million] people worldwide were living with HIV. Additionally, 1.8 million [1.4-2.4 million] new infections occurred, and 940,000 [670,000-1,300,000] people died from AIDS-related causes [1]. These estimates have declined substantially from peak estimates of 3.4 million [2.6-4.4 million] for new HIV infections in 1996, and 1.9 million [1.4-2.7 million] for AIDS-related deaths in 2004. However, despite tremendous progress in HIV prevention, care, and treatment that have attributed to decreasing numbers, challenges in achieving epidemic control persist. In particular, the number of new HIV infections is not declining fast enough: prevention efforts to avert new HIV infections need to be strengthened to match the number of HIV positive people placed on treatment [1]. Furthermore, focusing on populations that may be more vulnerable to HIV infection and identifying factors that are associated with infection in these populations must remain a public health priority.

In particular, military populations may be at higher risk for HIV. Service members are predominantly young, vulnerable to peer pressure, highly mobile, and often sent away for lengthy deployments [2, 3]. To alleviate stress and loneliness during deployments, studies have shown military personnel to engage in risky sexual behaviors (such as having sex with sex workers and multiple partners) during local or foreign deployments [4]. Other research has shown HIV prevalence to be substantially higher in some military forces compared to the general population $[5,6]$. Similar to the HIV policy in the US military [7], many militaries around the world also do not allow recruits who test HIV positive to enter service. Of concern is that military personnel are entering the forces HIV negative but are becoming HIV positive during their service. Characterizing key factors that place service members at higher risk for HIV infection during service is critical to develop an effective response to HIV.

Alcohol misuse has been identified as an important driver of the HIV epidemic in Sub-Saharan Africa. Meta analyses and systematic reviews have concluded that alcohol misuse is associated with an elevated risk of HIV infection by increasing risky sexual behaviors [8-13], such as sex with a sex worker, having multiple sexual partners, and participating in transactional sex. Engaging in sex with a female sex worker may increase the risk of HIV infection, as this group is disproportionally affected by HIV disease [14]. Multiple sexual partnerships may also elevate HIV risk as these relationships link individuals together and provide a pathway for the virus to move from one person to another. Additionally, the probability of exposure to primary HIV infection is amplified [15-17]. Transactional sex can also elevate HIV risk as individuals who engage in this behavior are more likely to experience different forms of abuse and violence $[18,19]$ or engage in other high risk sexual behaviors [20].

In military populations, several studies have shown rates of alcohol misuse to be higher among service members compared to the general population [21, 22]. Additionally, elevated odds of participating in risky sexual behaviors such as transactional sex, having multiple sexual partners, and having a higher number of sexual partners were observed among service members who exhibited patterns of excessive drinking [21, 23]. Alcohol misuse can lead to HIV infection through risky sexual behaviors, which ultimately impacts the health of service members, reduces operational readiness, and increases medical costs.

Several theories have been proposed to explain the associations between alcohol misuse and risky sexual behaviors. The alcohol myopia theory suggests that alcohol consumption can impair perception and thought, causing individuals to focus on cues that are most noticeable or important in the environment, while ignoring cues that are inhibitive or less salient as this would require more cognitive processing [24]. For example, in sexual situations, alcohol use may cause individuals to focus on immediate pleasure from the sexual act rather than the sexual partner's disease risk. Conversely, the alcohol expectancy theory proposes that the self-fulfilling expectations of the effects of alcohol use may influence the user's behavior [25]. For instance, those who believe alcohol use improves sexual performance and enhances sexual experiences may be more likely to consume alcohol while having sex. Furthermore, other studies have suggested that certain personality characteristics may predispose participation in risky sexual behaviors [26]. A study found that personality traits of sensation seeking and impulsive decision making are correlated with sexual risk taking [26, 27].

The purpose of this study was to estimate the prevalence of probable problematic alcohol use in comparison to no/low-risk alcohol use and identify associated factors in the Armed Forces of the Democratic Republic of the Congo (FARDC). Other objectives include examining associations between probable problematic alcohol use with the following risky sexual behaviors: sex with a sex worker, having multiple sexual partners, and participation in transactional sex. This study aimed to contribute to the paucity of research on alcohol use and risky sexual behaviors among military populations in SubSaharan Africa.

\section{Methods}

\section{Study description and sampling}

From October 2013 to April 2014, a seroprevalence and behavioral epidemiology risk survey (SABERS) was conducted in the FARDC [28]. The purpose of the SABERS 
was to determine the prevalence of HIV and syphilis and identify associated demographics and risk behaviors. Active duty men and women who were 18 years of age or older were eligible to participate in the cross-sectional study. To obtain a representative sample, 30 military bases from nine military regions were selected for study participation based on logistical feasibility. From these bases, military units were randomly selected for inclusion. The target sample size of the SABERS was proportional to the overall military size by rank. Convenience sampling was used to increase representation of senior officers (i.e., Captain, Major, and Colonel) as these individuals were typically affected by other competing military interests (i.e., prior commitments) that made random sampling difficult. Therefore, all available senior officers from the selected military units were invited to participate in the informational briefing. All other lower ranked service members were asked to draw a piece of paper from a hat - those who selected a marked piece of paper were invited to participate in the informational briefing. At the briefing, the study purpose, procedures, risks, and benefits were explained by a study team member. To ensure participants understood all aspects of the study, the consent form was available in either French or Lingala (a local dialect). All participants had the right to accept or decline participation in the SABERS and could stop the survey at any time without any negative consequences. All participants were given an opportunity to ask questions prior to giving electronic informed consent on a computer tablet. Compensation was not provided for participation in the study. Those who consented to take part in this study completed a computer-assisted personal interview performed by a trained study counselor in a private setting, and were then tested for HIV and syphilis.

The original research was conducted in compliance with all federal and local regulations and was approved by institutional review boards (IRBs) in San Diego, CA, USA (Naval Health Research Center [NHRC]) and Kinshasa, Democratic Republic of the Congo (Ministère de l'Enseignement Supérieur, Universitaire et Recherche Scientifique, Université de Kinshasa). Approval for secondary data analysis was obtained by the NHRC IRB.

\section{Variables and measures}

The questionnaire was developed by epidemiologists at the United States Department of Defense HIV/AIDS Prevention Program and was adapted from similar studies conducted in other African militaries. Input from FARDC leadership was solicited and incorporated into the final survey, as well as feedback from FARDC service members who pretested the questionnaire for comprehension and cultural sensitivities prior to study implementation. The questionnaire was designed to collect information on demographics and HIV-related risk behaviors.

Demographic variables of interest included age, marital status, and education level. Marital status was categorized as single/widowed/divorced, single and living with a partner, or monogamous/polygamous marriage. Participants were asked to report the highest education level they completed. Responses were grouped as no school/ completed less than primary, completed primary, completed secondary, or completed high school or university. Military characteristics of interest included rank, branch, and local deployments of more than six months in the past two years. Rank was categorized as recruit/ soldier/corporal, non-commissioned officer, or officer. Military branch included the Army, Marine, Airforce, or other.

Hazardous, harmful, and dependent alcohol use was measured with the Alcohol Use Disorders Identification Test (AUDIT) [29]. The AUDIT was developed by researchers at the World Health Organization (WHO) and validated across different populations in six countries. It has been shown to accurately assess patterns of problematic alcohol use across age, gender, and cultures. WHO researchers defined hazardous alcohol use as a pattern of alcohol consumption that increased the risk of harmful consequences (e.g., physical, mental, or social) for the user or others. Harmful alcohol use was defined as a pattern of alcohol consumption that caused physical or mental damage to the user's health and alcohol dependence referred to a cluster of behavioral, cognitive, and physiological symptoms that may develop after repeated use. These include a strong desire to consume alcohol, impaired control over its use, and a higher priority given to alcohol consumption compared to other activities [29]. The AUDIT consisted of 10 questions asking about recent alcohol use, alcohol dependence symptoms, and alcohol-related problems. A total score was computed from the questions which ranged from 0 to 40 and reflected a person's level of risk related to alcohol. A score of 0 was an indicator of no alcohol use, 1 to 7 an indicator of low-risk alcohol use, 8 to 19 an indicator of probable hazardous and harmful alcohol use, and 20 or higher an indicator of possible alcohol dependence. In this study, WHO's recommended cut-point of 8 for the AUDIT scale was used to identify individuals exhibiting potential alcohol problems: participants with scores of 8 or higher (defined as probable problematic alcohol use) were compared to those with scores of 7 or lower (defined as no/low-risk alcohol use).

Participants provided information on having sex with a sex worker, having multiple sexual partners, and engaging in transactional sex. Sex with a sex worker in the past 12 months was defined as sex with any person who provided sex in exchange for money. Multiple sexual 
partners was defined as participants who reported ever having sex with more than one sexual partner in the same week. Transactional sex was defined as ever paying or receiving money, shelter, food, drugs, favors or gifts in exchange for sex with a partner who was not a sex worker.

Participants also reported the influence of alcohol use on unintended sex and condom use in the past three months with the following questions: "In the last three months, did drinking alcohol influence your decision about or prevent you from using condoms or using condoms correctly? and "In the last three months, did you ever have unintended sex as a result of drinking alcohol?"

\section{Statistical analysis}

Frequencies and percentages for categorical variables and means and standard deviations for continuous variables were computed. Bivariate logistic regression models were used to examine associations of demographic and military characteristics (i.e., categorical age, marital status, education level, rank, branch, and local deployment) with alcohol use. Several multivariable logistic regression models were used to independently examine the associations of alcohol use with sex with a sex worker, multiple sexual partners, and transactional sex after adjusting for categorical age, marital status, and education level. These variables were included as potential confounders based on previous literature [30, 31]. The multivariable models were restricted to those who reported ever having sexual intercourse which was defined as vaginal and/or anal sex, but did not include oral sex. Collinearity was assessed by evaluating tolerance values and the goodness-of-fit of the models was examined through Hosmer-Lemoshow tests. All tests were two-tailed, with $p<0.05$ indicating statistical significance. Data analysis was conducted using SAS software, version 9.4 (SAS Institute Inc., Cary, NC, USA).

\section{Results}

Of 2840 service members who were invited to the informational briefing, 2816 (99.2\%) met eligibility criteria and consented to participate. Of these participants, 2751 (97.7\%) completed the survey and biological testing. Due to the small number of women $(n=140)$, analyses were restricted to 2611 active duty male service members. Male service members who did not report ever having sexual intercourse $(n=61)$ or were missing data on participation in transactional sex $(n=1)$ were further excluded, resulting in a final sample size of 2549 men for the current study.

The mean age of these participants was 41.5 years (standard deviation $=10.3$; range $=19$ to 83 ) and the majority $(40.0 \%)$ were in the 30 to 39 age group. Most men were married (either monogamous or polygamous) and had completed at least primary school (78.1 and 50.7\%, respectively). With regards to military characteristics, the majority were ranked as a non-commissioned officer (41.4\%) or officer (43.9\%), and were serving in the Army (66.5\%). Almost a quarter $(20.2 \%)$ of respondents reported they had been on a local deployment lasting at least six months within the past two years. Fifteen percent $(14.9 \%)$ of men screened positive for probable problematic alcohol use (AUDIT score $\geq 8$ ) (Table 1).

Several variables were significantly associated with probable problematic alcohol use (Table 2). The odds of probable problematic alcohol use were elevated for men

Table 1 Characteristics of male FARDC service members ( $N=$ 2549)

\begin{tabular}{|c|c|c|}
\hline \multirow{2}{*}{$\begin{array}{l}\text { Characteristic } \\
\text { Age in years, mean (standard deviation) }\end{array}$} & \multicolumn{2}{|c|}{$\begin{array}{l}\text { Total } \\
(N=2549)\end{array}$} \\
\hline & 41.5 & $(10.3)$ \\
\hline \multicolumn{3}{|l|}{ Age group, $n(\%)$} \\
\hline $19-29$ & 239 & (9.4) \\
\hline $30-39$ & 1019 & $(40.0)$ \\
\hline $40-49$ & 759 & $(29.8)$ \\
\hline $50+$ & 532 & $(20.8)$ \\
\hline \multicolumn{3}{|l|}{ Marital status, $n(\%)$} \\
\hline Single/widowed/divorced & 203 & (8.0) \\
\hline Single and living with a partner & 353 & $(13.9)$ \\
\hline Monogamous/polygamous marriage & 1993 & $(78.1)$ \\
\hline \multicolumn{3}{|l|}{ Educational attainment, $n$ (\%) } \\
\hline No school/completed less than primary & 358 & $(14.0)$ \\
\hline Completed primary & 1291 & $(50.7)$ \\
\hline Completed secondary & 663 & $(26.0)$ \\
\hline Completed high school or university & 237 & $(9.3)$ \\
\hline \multicolumn{3}{|l|}{ Military rank, $n(\%)$} \\
\hline Recruit/soldier/corporal & 376 & $(14.7)$ \\
\hline Non-commissioned officer & 1055 & $(41.4)$ \\
\hline Officer & 1118 & $(43.9)$ \\
\hline \multicolumn{3}{|l|}{ Military branch, $n(\%)$} \\
\hline Army & 1696 & $(66.5)$ \\
\hline Marine & 122 & $(4.8)$ \\
\hline Airforce & 54 & $(2.1)$ \\
\hline Other & 677 & $(26.6)$ \\
\hline \multicolumn{3}{|l|}{ Local deployment, $n$ (\%) } \\
\hline Yes & 516 & $(20.2)$ \\
\hline No & 2033 & $(79.8)$ \\
\hline \multicolumn{3}{|l|}{ AUDIT score } \\
\hline 0 to 7 : no/low-risk alcohol use & 2169 & $(85.1)$ \\
\hline 8 or higher: probable problematic alcohol use & 380 & $(14.9)$ \\
\hline
\end{tabular}

AUDIT: Alcohol Use Disorders Identification Test 
Table 2 Factors associated with probable problematic alcohol use among male FARDC service members $(N=2549)$

\begin{tabular}{|c|c|c|c|c|}
\hline & $\begin{array}{l}\text { Probable problematic alcohol use } \\
(n=380)\end{array}$ & $\begin{array}{l}\text { No/low-risk alcohol use } \\
(n=2169)\end{array}$ & & \\
\hline Characteristic & n (\%) & $n(\%)$ & OR $(95 \% \mathrm{Cl})$ & $P$ value \\
\hline Age group & & & & $<0.0001$ \\
\hline $50+$ & $50(13.2)$ & $482(22.2)$ & ref $(1.0)$ & \\
\hline $40-49$ & 119 (31.3) & $640(29.5)$ & $1.79(1.26-2.55)$ & \\
\hline $30-39$ & $187(49.2)$ & $832(38.4)$ & $2.17(1.56-3.02)$ & \\
\hline $19-29$ & $24(6.3)$ & $215(9.9)$ & $1.08(0.65-1.80)$ & \\
\hline Marital status & & & & 0.0015 \\
\hline Monogamous/polygamous marriage & $271(71.3)$ & $1722(79.4)$ & ref $(1.0)$ & \\
\hline Single and living with a partner & $73(19.2)$ & $280(12.9)$ & $1.66(1.24-2.21)$ & \\
\hline Single/widowed/divorced & $36(9.5)$ & $167(7.7)$ & $1.37(0.94-2.01)$ & \\
\hline Educational attainment & & & & 0.0804 \\
\hline Completed high school or university & $27(7.1)$ & $210(9.7)$ & ref (1.0) & \\
\hline Completed secondary & $93(24.5)$ & $570(26.3)$ & $1.27(0.80-2.00)$ & \\
\hline Completed primary & $193(50.8)$ & $1098(50.6)$ & $1.37(0.89-2.10)$ & \\
\hline No school/completed less than primary & $67(17.6)$ & $291(13.4)$ & $1.79(1.11-2.90)$ & \\
\hline Military rank & & & & 0.0180 \\
\hline Officer & $145(38.3)$ & $973(44.9)$ & ref $(1.0)$ & \\
\hline Non-commissioned officer & $182(47.8)$ & $873(40.2)$ & $1.40(1.10-1.77)$ & \\
\hline Recruit/soldier/corporal & $53(13.9)$ & $323(14.9)$ & $1.10(0.79-1.55)$ & \\
\hline Military branch & & & & 0.1709 \\
\hline Army & $240(63.2)$ & $1456(67.1)$ & ref $(1.0)$ & \\
\hline Marine & $14(3.7)$ & $108(5.0)$ & $0.79(0.44-1.40)$ & \\
\hline Airforce & $9(2.4)$ & $45(2.1)$ & $1.21(0.59-2.51)$ & \\
\hline Other & $117(30.7)$ & $560(25.8)$ & $1.27(0.99-1.61)$ & \\
\hline Local deployment & & & & 0.4006 \\
\hline No & $297(78.2)$ & $1736(80.0)$ & ref $(1.0)$ & \\
\hline Yes & $83(21.8)$ & $433(20.0)$ & $1.12(0.86-1.46)$ & \\
\hline
\end{tabular}

SD: Standard deviation; OR: Odds ratio; $\mathrm{Cl}$ : Confidence interval

in the 30-39 and 40-49 age groups compared to men 50 or older (odds ratio [OR] 30 -39 age group $=2.17$; $95 \%$ confidence interval $[\mathrm{CI}]=1.56-3.02$; OR 40-49 age group $=1.79 ; 95 \% \mathrm{CI}=1.26-2.55$ ). The odds of probable problematic alcohol use were significantly higher among participants who were single and living with a partner compared to those who were in a monogamous or polygamous marriage $(\mathrm{OR}=1.66$; $95 \% \mathrm{CI}=1.24-2.21)$. The odds were also elevated for those who were ranked as a non-commissioned officer compared to an officer (OR = 1.40 ; $95 \% \mathrm{CI}=1.10-1.77)$. No statistically significant associations were observed for education level, branch or local deployment (all $p>0.05$ ).

With regards to risky sexual behaviors, $12.2 \%$ of all participants reported sex with a sex worker, $33.8 \%$ indicated having multiple sexual partners, and $35.2 \%$ participated in transactional sex. All of these behaviors were more commonly reported among participants with probable problematic alcohol use compared to those with no/low-risk alcohol use (Table 3).

Probable problematic alcohol use was found to be significantly associated with sex with a sex worker, multiple sexual partners, and transactional sex (Table 4). After adjusting for age, marital status, and education level, the odds of having sex with a sex worker were two times higher among men with probable problematic alcohol use compared to men with no/low-risk alcohol use (adjusted odds ratio $[\mathrm{aOR}]=2.36 ; 95 \% \mathrm{CI}=1.78-3.13$ ). The odds of having multiple sexual partners and participating in transactional sex were also elevated among men with probable problematic alcohol use compared to men with no/low-risk alcohol use (aOR multiple sexual partners $=2.08$; 95\% CI $=1.66-$ 2.60; aOR transactional sex $=1.99 ; 95 \% \mathrm{CI}=1.59-2.50$ ). Results from the Hosmer-Lemeshow tests indicated a good fit for each model. Additionally, multicollinearity was not an issue. 
Table 3 Risky sexual behaviors by alcohol use among male FARDC service members $(N=2549)$

\begin{tabular}{llll}
\hline & $\begin{array}{l}\text { Total } \\
(N=2549)\end{array}$ & $\begin{array}{l}\text { Probable problematic alcohol use } \\
(n=380)\end{array}$ & $\begin{array}{l}\text { No/low-risk alcohol use } \\
(n=2169)\end{array}$ \\
\hline $\begin{array}{l}\text { Risky Sexual Behavior } \\
\text { Sex with a sex worker }\end{array}$ & $\mathrm{n}(\%)$ & $292(76.8)$ & $\mathrm{n}(\%)$ \\
$\quad$ No & $2237(87.8)$ & $88(23.2)$ & $1945(89.7)$ \\
$\quad$ Yes & $312(12.2)$ & & $224(10.3)$ \\
Multiple sexual partners & & $192(50.5)$ & $1495(68.9)$ \\
$\quad$ No & $1687(66.2)$ & $188(49.5)$ & $674(31.1)$ \\
$\quad$ Yes & $862(33.8)$ & & $1463(67.5)$ \\
Participated in transactional sex* & & $189(49.7)$ & $706(32.5)$ \\
No & $1652(64.8)$ & $191(50.3)$ & \\
$\quad$ Yes & $897(35.2)$ & & \\
\hline
\end{tabular}

* Paid or received money, shelter, food, drugs, favors, or gifts in exchange for sex

Of participants who had sex and drank alcohol in the past three months $(n=1209), 14.3 \%(n=173)$ reported alcohol consumption influenced their decision about using condoms or prevented them from using condoms correctly. A total of $18.4 \%(n=223)$ also reported unintended sex as a result of drinking alcohol (results not shown in a table).

\section{Discussion}

There is mounting evidence suggesting that alcohol use is an important factor to address in the HIV epidemic. Previous research has shown that patterns of problematic alcohol consumption are more likely to occur in regions that also have the highest burden of HIV disease, notably in eastern and southern Africa [32]. With projections that the alcohol market is anticipated to flourish substantially in this region due to growing economies and urbanization [33], identification of priority populations, such as militaries, who may be at higher risk for alcohol misuse is highly important. This study identified factors associated with probable problematic alcohol use in male FARDC service

Table 4 Adjusted associations of probable problematic alcohol use with risky sexual behaviors among male FARDC service members $(N=2549)$

\begin{tabular}{lll}
\hline Risky Sexual Behaviors & AOR $^{*}(95 \% \mathrm{Cl})$ & $P$ value \\
\hline $\begin{array}{l}\text { Had sex with a sex worker } \\
\text { Yes vs. No }\end{array}$ & $2.36(1.78-3.13)$ & $<0.0001$ \\
$\begin{array}{l}\text { Had multiple sexual partners } \\
\text { Yes vs. No }\end{array}$ & $<0.0001$ \\
$\begin{array}{l}\text { Participated in transactional sex** } \\
\text { Yes vs. No }\end{array}$ & $1.99(1.59-2.50)$ & $<0.0001$ \\
$\begin{array}{l}\text { AOR: adjusted odds ratio. } \\
\text { *Adjusted for age, marital status, and education level } \\
\text { ** Paid or received money, shelter, food, drugs, favors, or gifts in exchange } \\
\text { for sex }\end{array}$
\end{tabular}

members and evaluated associations of misuse with risky sexual behaviors.

A considerable proportion (14.9\%) of male service members in the FARDC screened positive for probable problematic alcohol use. This estimate is almost two times higher than rates reported among men in the general population of the Democratic Republic of the Congo (9.1\%). These findings are comparable to other studies that have also found alcohol misuse to be substantially higher among military populations compared to their civilian counterparts [21, 22]. There are several possible explanations for higher rates of alcohol misuse among service members. Historically, alcohol use has been a part of military culture as a way to alleviate stress [3436]. Service members in the FARDC may also have more disposable income than their civilian counterparts, which may be used to purchase alcohol. Additionally, there are unique circumstances of military service that may be associated with problematic drinking including exposure to combat and traumatic events [37-40] and frequent and lengthy deployments from loved ones [21]. Comprehensive measures must be taken to reduce alcohol misuse among male FARDC service members. Military institutions have the ability to implement policies that can change norms around alcohol use. For example, they can refuse to sell alcohol on base, or limit its sale to certain hours or days of the week. Military health systems can also include screening for problematic alcohol use during annual health screens to determine fitness for duty. Men who screen positive for probable problematic alcohol use should be referred to a military or civilian medical center for treatment. Furthermore, social marketing campaigns can take advantage of perceptions that military members are 'tough' to promote messages that unsafe alcohol use may interfere with physical fitness.

Several demographic and military factors were found to be correlated with probable problematic alcohol use 
including being single and living with a partner, which is consistent with previous studies in other militaries [21, 41-43]. Post-hoc analyses found that men who were single and living with a partner were substantially younger than men who were married (mean age of 35.8 years old vs. mean age of 43.1 years old). These participants may have less responsibilities (e.g., no children) and therefore may be more likely to have free time to engage in social activities that involve drinking. Higher rank (i.e., rank of noncommissioned officer) was also associated with probable problematic alcohol use. The FARDC should hold a workshop with higher leadership to address alcohol misuse and its detrimental effects on the individual and overall force health. The FARDC should also draft a policy on alcohol management and distribute this policy to higher leadership. Having senior leaders lead by example may have a positive outcome on service members they are responsible for overseeing. Age was also associated with alcohol misuse. The odds of probable problematic alcohol use were highest among men who were in the 30 to 49 age groups. While previous studies have shown alcohol misuse to be a problem among service members 17 to 25 years of age [41] and those 20 to 29 [42, 43], this study demonstrated that probable problematic alcohol use could still be an issue among older age groups. In summary, these findings suggest that certain characteristics place military personnel at increased risk for probable problematic alcohol use. Reducing and preventing dangerous alcohol consumption must be a priority for FARDC leadership to maintain operational readiness. Comprehensive alcohol misuse education would benefit all forces, but efforts should be targeted at men who are 30 to 49 years of age, not married, and of higher rank.

Probable problematic alcohol use was found to be associated with having sex with a sex worker, engaging in multiple sexual partners, and participating in transactional sex among male service members in the FARDC. These findings are consistent with previous literature conducted in other military populations [21, 44]. Furthermore, a substantial percentage of participants reported that alcohol had a direct effect on their sexual behaviors: $14.3 \%$ reported alcohol consumption influenced their decision about using condoms or prevented them from using condoms correctly and $18.4 \%$ reported alcohol use led to unintended sex. While the FARDC does have a comprehensive HIV prevention program, these data highlight the need to incorporate alcohol abuse prevention into the existing program and underscore its role in HIV transmission and acquisition through risky sexual behaviors. Additionally, other preventive measures include the promotion of social events that don't involve alcohol consumption (e.g., participation in sports).
Several study limitations are noted. All data were self-reported and subject to recall and social desirability bias. However, interviewers were trained in proper data collection techniques, and all respondents were instructed they could stop the survey at any time or not answer any questions they did not feel comfortable providing a response. Comparisons of probable problematic alcohol use in the military and civilian counterparts should be interpreted with caution as different measurements for alcohol misuse may have been used: the AUDIT was used in this study, but it is unclear what measurement was used in the WHO global report on alcohol use [45]. Due to the crosssectional nature of the study, temporality and causality between variables cannot be determined. While strong associations between probable problematic alcohol use and risky sexual behaviors were observed, temporality and the causal relationship remains inconclusive for various reasons. Residual confounding is plausible as other factors (e.g., personality traits and mental health problems) that may affect these associations were not measured in the SABERS. Reverse causality is plausible as well; participants may have used alcohol as a mechanism to cope with participation in risky sexual behaviors. Further studies are needed to elucidate the causal relation between alcohol use and risky sexual behaviors. Convenience sampling of senior officers may have introduced selection bias into the SABERS study and results should be interpreted with this limitation in mind. Due to the small number of women who participated in the original SABERS study, analyses among female service members were not performed and therefore study results are not generalizable to this group. Further studies among female service members are needed to characterize the relation between alcohol use and risky sexual behaviors.

\section{Conclusions}

These data add to growing research among African military populations that alcohol use may fuel the HIV epidemic by increasing risky sexual behaviors. Identifying specific subsets of the FARDC population who are more likely to misuse alcohol is an important first step for FARDC leadership to implement a comprehensive alcohol misuse prevention program that is tailored to those at highest risk. Preventing alcohol abuse, screening for problematic use, and referring those who have exhibited patterns of excessive drinking to medical care and treatment are critical activities to incorporate into existing HIV prevention programs. Additionally, developing effective messages and interventions to mitigate harmful and hazardous alcohol use in relation to risky sexual behaviors are essential to maintain troop health and preserve force readiness. 


\section{Abbreviations}

AUDIT: Alcohol Use Disorders Identification Test; DHAPP: Department of Defense HIV/AIDS Prevention Program; FARDC: Armed Forces of the Democratic Republic of the Congo; IRB: Institutional Review Board; NCO : Non-commissioned officer; NHRC: Naval Health Research Center; SABERS: Seroprevalence and Behavioral Epidemiology Risk Survey; SW: Sex worker; WHO: World Health Organization

\section{Acknowledgements}

We extend our sincere appreciation to the military members of the Armed Forces of the Democratic Republic of the Congo who participated in this study and to military staff who helped bring this study to fruition. Thank you to Metabiota for their tremendous support in this study.

\section{Disclaimer}

I am a military service member (or employee of the U.S. Government). This work was prepared as part of my official duties. Title 17 U.S.C. § 105 provides the "Copyright protection under this title is not available for any work of the United States Government." Title 17 U.S.C. § 101 defines a U.S. Government work as work prepared by a military service member or employee of the U.S. Government as part of that person's official duties. The views expressed in this article are those of the authors and do not necessarily reflect the official policy or position of the Department of the Navy, Department of the Army, Department of the Air Force, Department of Veterans Affairs, Department of Defense, or the U.S. Government. Approved for public release; distribution unlimited.

\section{Authors' contributions}

BRT came up with the conception of the work, analyzed and interpreted the data, and was a major contributor in writing and revising the manuscript. $A D$ was involved in the conception and design of the original study and contributed to the analysis and writing of the manuscript. MS contributed substantially to the writing and revision of the manuscript. CM contributed substantially to the revision of the manuscript. AMM and GKK were involved in the acquisition of data for the original study and contributed substantially to the drafting and revision of the manuscript. All authors have read and approved the final manuscript

\section{Funding}

This research is supported by the Department of Defense HIV/AIDS Prevention Program. Funding for the original study was provided by the President's Emergency Plan for AIDS Relief (PEPFAR) and funding for secondary data analysis was provided by Uniformed Services University of the Health Sciences (USUHS): award number HU0001-17-1-0045. The funders did not have any additional role in the design of the study, collection, analysis, interpretation of the data, nor writing or preparation of the manuscript.

\section{Availability of data and materials}

The datasets generated and/or analyzed during the current study are not publicly available due to the sensitive and confidential nature of the data as determined by the HIV/AIDS Directorate of the Armed Forces of the Democratic Republic of the Congo. Requests for data access should be made to Colonel Anthony Mbuyi (mbuyimutombeanthony@yahoo.com) and Bonnie Tran (bonnie.r.tran.ctr@mail.mil).

\section{Ethics approval and consent to participate}

Human subjects participated in this study after giving their free and informed consent. Each subject indicated their consent to participate or not participate by checking a box for "I consent" or "No, I do not consent" on an electronic computer tablet. This research has been conducted in compliance with all applicable federal regulations governing the protection of human subjects in research in the US (Naval Health Research Center Institutional Review Board; Protocol NHRC.2013.0024) and the Democratic Republic of the Congo (Ministère de l'Enseignement Supérieur, Universitaire et Recherche Scientifique, Université de Kinshasa). The use of electronic informed consent was approved by both ethics committees.

\section{Consent for publication}

Not applicable.

\section{Competing interests}

The authors declare that they have no competing interests.

\section{Author details}

'Department of Defense HIV/AIDS Prevention Program, San Diego, California, USA. ${ }^{2}$ Leidos Inc, Reston, Virginia, USA. ${ }^{3}$ Armed Forces of the Democratic Republic of the Congo, Kinshasa, Democratic Republic of the Congo.

Received: 9 May 2019 Accepted: 16 October 2019

Published online: 28 October 2019

\section{References}

1. Joint United Nations Programme on HIV/AIDS (UNAIDS). UNAIDS DATA 2018. UNAIDS. 2018. Available from: https://www.who.int/hiv/pub/arv/ global-aids-update-2016-pub/en/. .

2. Miller N, Yeager R. By virtue of their occupation, soldiers and sailors are at greater risk. Special report: the military. AIDS Anal Afr. 1995;5(6):8-9.

3. Joint United Nations Programme on HIV/AIDS (UNAIDS). AIDS and the Military: UNAIDS Point of View. Geneva: UNAIDS; 1998.

4. Nwokoji UA, Ajuwon AJ. Knowledge of AIDS and HIV risk-related sexual behavior among Nigerian naval personnel. BMC Public Health. 2004;4:24.

5. Djibo DA, Sahr F, Allen MJ, Jain S, Araneta MRG, Brodine SK, et al. Prevalence and risk factors for human immunodeficiency virus (HIV) and syphilis infections among military personnel in Sierra Leone. Curr HIV Res. 2017;15:128-36

6. Rimoin AW, Hoff NA, Djoko CF, Kisalu NK, Kashamuka M, Tamoufe U, et al. HIV infection and risk factors among the armed forces personnel stationed in Kinshasa, Democratic Republic of Congo. Int J STD AIDS. 2015;26(3):187-95.

7. Department of Defense (DoD). DoD Human Immunodeficiency Virus (HIV) Testing Program and Drug and Alcohol Testing (DAT) Program. DoD. 2018. Available from: https:/www.mepcom.army.mil/Portals/112/Documents/ PubsForms/Regs/r-0040-008.pdf.

8. Woolf-King SE, Maisto SA. Alcohol use and high-risk sexual behavior in subSaharan Africa: a narrative review. Arch Sex Behav. 2011;40(1):17-42.

9. Kalichman SC, Simbayi LC, Kaufman M, Cain D, Jooste S. Alcohol use and sexual risks for HIV/AIDS in sub-Saharan Africa: systematic review of empirical findings. Prev Sci. 2007;8(2):141-51.

10. Shuper PA, Neuman M, Kanteres F, Baliunas D, Joharchi N, Rehm J. Causal considerations on alcohol and HIV/AIDS--a systematic review. Alcohol Alcohol. 2010;45(2):159-66.

11. Baliunas D, Rehm J, Irving H, Shuper P. Alcohol consumption and risk of incident human immunodeficiency virus infection: a meta-analysis. Int J Public Health. 2010:55(3):159-66.

12. Fisher JC, Bang H, Kapiga SH. The association between HIV infection and alcohol use: a systematic review and meta-analysis of African studies. Sex Transm Dis. 2007;34(11):856-63.

13. Cook RL, Clark DB. Is there an association between alcohol consumption and sexually transmitted diseases? A systematic review. Sex Transm Dis. 2005;32(3):156-64.

14. Baral S, Beyrer C, Muessig K, Poteat T, Wirtz AL, Decker MR, et al. Burden of HIV among female sex workers in low-income and middle-income countries: a systematic review and meta-analysis. Lancet Infect Dis. 2012; 12(7):538-49.

15. Drumright LN, Gorbach PM, Holmes KK. Do people really know their sex partners? Concurrency, knowledge of partner behavior, and sexually transmitted infections within partnerships. Sex Transm Dis. 2004;31(7): 437-42.

16. Mah TL, Halperin DT. Concurrent sexual partnerships and the HIV epidemics in Africa: evidence to move forward. AIDS Behav. 2010;14(1):11-16; dicussion 34-7.

17. Morris M, Kretzschmar M. Concurrent partnerships and the spread of HIV. AIDS. 1997;11(5):641-8.

18. Choudhry V, Ambresin AE, Nyakato VN, Agardh A. Transactional sex and HIV risks - evidence from a cross-sectional national survey among young people in Uganda. Glob Health Action. 2015;8:27249.

19. Zembe YZ, Townsend L, Thorson A, Silberschmidt M, Ekstrom AM. Intimate partner violence, relationship power inequity and the role of sexual and social risk factors in the production of violence among young women who have multiple sexual Partners in a Peri-Urban Setting in South Africa. PLoS One. 2015;10(11):e0139430. 
20. Okigbo CC, McCarraher DR, Chen M, Pack A. Risk factors for transactional sex among young females in post-conflict Liberia. Afr J Reprod Health. 2014;18(3):133-41.

21. Tran BR, Davis A, Ito SI, Matchere F, Reader E, Nkhoma V, et al. Alcohol and Cannabis use and sexual risk behaviors in the Malawi Defence force. AIDS Behav. 2018;22(9):2851-60.

22. Tran BR, Thomas AG, Ditsela M, Vaida F, Phetogo R, Kelapile D, et al. Condom use behaviours and correlates of use in the Botswana Defence force. Int J STD AIDS. 2013;24(11):883-92.

23. Bing EG, Ortiz DJ, Ovalle-Bahamon RE, Cheng KG, Huang FH, Ernesto F, et al. HIV/AIDS behavioral surveillance among Angolan military men. AIDS Behav. 2008;12(4):578-84.

24. Steele CM, Josephs RA. Alcohol myopia. Its prized and dangerous effects. Am Psychol. 1990;45(8):921-33.

25. Crowe LC, George WH. Alcohol and human sexuality: review and integration. Psychol Bull. 1989;105(3):374-86

26. Kalichman SC, Weinhardt L, DiFonzo K, Austin J, Luke W. Sensation seeking and alcohol use as markers of sexual transmission risk behavior in HIVpositive men. Ann Behav Med. 2002;24(3):229-35.

27. Donohew L, Zimmerman R, Cupp PS, Novak S, Colon S, Ritta A. Sensation seeking, impulsive decision-making, and risky sex: implications for risk-taking and design of interventions. Personal Individ Differ. 2000;28(6):1079-91.

28. Macera CA, Ito SI, Hale BR, Shaffer RA, Thomas AG, Dickieson J. Conducting HIV Seroprevalence and behavioral epidemiology risk surveys (SABERS) among partner military populations. Curr HIV Res. 2017;15(2):82-9.

29. Babor T.F., Higgins-Biddle J.C., Saunders J.B., Monteiro M.G. The Alcohol Use Disorders Identification Test (AUDIT): Guidelines for Use in Primary Care. World Health Organization. 2001. Available from: https://www.who.int/ substance_abuse/publications/audit/en/. .

30. Simbayi LC, Kalichman SC, Cain D, Cherry C, Jooste S, Mathiti V. Alcohol and risks for HIV/AIDS among sexually transmitted infection clinic patients in Cape Town. South Africa Subst Abus. 2006;27(4):37-43.

31. Weiser SD, Leiter K, Heisler M, McFarland W, Percy-de Korte F, DeMonner SM, et al. A population-based study on alcohol and high-risk sexual behaviors in Botswana. PLoS Med. 2006;3(10):e392.

32. Fritz K, Morojele N, Kalichman S. Alcohol: the forgotten drug in HIV/AIDS. Lancet. 2010;376(9739):398-400.

33. Toesland F. Alcohol consumption rising in Africa despite obstacles. African Business. 12 Jan 2016. Available from: https://africanbusinessmagazine.com/ sectors/retail/alcohol-consumption-rising-in-africa-despite-obstacles/. .

34. Jones E, Fear NT. Alcohol use and misuse within the military: a review. Int Rev Psychiatry. 2011;23(2):166-72.

35. Pack A. Nelson's blood: the story of naval rum. Annapolis: Naval Institute Press; 1983.

36. Poehlman JA, Schwerin MJ, Pemberton MR, Isenberg K, Lane ME, Aspinwall K. Socio-cultural factors that foster use and abuse of alcohol among a sample of enlisted personnel at four navy and marine corps installations. Mil Med. 2011;176(4):397-401.

37. Jacobson IG, Ryan MA, Hooper TI, Smith TC, Amoroso PJ, Boyko EJ, et al. Alcohol use and alcohol-related problems before and after military combat deployment. Jama. 2008;300(6):663-75.

38. Milliken CS, Auchterlonie JL, Hoge CW. Longitudinal assessment of mental health problems among active and reserve component soldiers returning from the Iraq war. Jama. 2007;298(18):2141-8.

39. Cheng KG, Ortiz DJ, Weiss RE, Shi L, Ovalle-Bahamon RE, Ernesto F, et al. Patterns of alcohol consumption and factors influencing problematic drinking among Angolan soldiers. J Subst Use. 2012;17(2):138-49.

40. Kehle SM, Ferrier-Auerbach AG, Meis LA, Arbisi PA, Erbes CR, Polusny MA. Predictors of postdeployment alcohol use disorders in National Guard soldiers deployed to operation Iraqi freedom. Psychol Addict Behav. 2012; 26(1):42-50.

41. Bray RM, Marsden ME, Guess LL, Herbold JR. Prevalence, trends, and correlates of alcohol use, nonmedical drug use, and tobacco use among U. S. military personnel. Mil Med. 1989;154(1):1-11.

42. Waller M, McGuire AC, Dobson AJ. Alcohol use in the military: associations with health and wellbeing. Subst Abuse Treat Prev Policy. 2015;10:27.

43. Fear NT, Iversen A, Meltzer H, Workman L, Hull L, Greenberg N, et al. Patterns of drinking in the UK armed forces. Addiction. 2007;102(11):1749-59.

44. Tavarez Ml, Chun H, Anastario MP. Correlates of sexual risk behavior in sexually active male military personnel stationed along border-crossing zones in the Dominican Republic. Am J Mens Health. 2011;5(1):65-77.
45. World Health Organization (WHO). Global status report on alcohol and health. WHO. 2018:2018. Available from: https://www.who.int/substance_ abuse/publications/global_alcohol_report/gsr_2018/en/.

\section{Publisher's Note}

Springer Nature remains neutral with regard to jurisdictional claims in published maps and institutional affiliations.
Ready to submit your research? Choose BMC and benefit from:

- fast, convenient online submission

- thorough peer review by experienced researchers in your field

- rapid publication on acceptance

- support for research data, including large and complex data types

- gold Open Access which fosters wider collaboration and increased citations

- maximum visibility for your research: over $100 \mathrm{M}$ website views per year

At $\mathrm{BMC}$, research is always in progress.

Learn more biomedcentral.com/submissions 